\title{
A IMAGEM COMO FERRAMENTA NA PESQUISA QUALITATIVA EM SAÚDE: RELATO DE EXPERIÊNCIA
}

\section{IMAGE AS A TOOL IN QUALITATIVE RESEARCH IN HEALTH: EXPERIENCE REPORT}

\author{
Elaine de Oliveira Souza Fonseca ${ }^{1}$ \\ Larissa Chaves Pedreira ${ }^{2}$ \\ Ana Isabel Rodrigues ${ }^{3}$ \\ Roberta Góes Pereira ${ }^{4}$ \\ Nildete Pereira Gomes ${ }^{5}$
}

\begin{abstract}
Resumo: A imagem é uma relevante ferramenta em pesquisas qualitativas. Este trabalho objetivou descrever a experiência do uso da imagem como método de coleta de dados por meio da Técnica de Associação Livre de Palavras, em um estudo sobre cuidados em saúde bucal de idosos hospitalizados. A pesquisa ocorreu em um hospital universitário situado em Salvador, Bahia, Brasil, tendo a equipe de enfermagem como colaboradora do estudo. Por se tratar de pesquisa com seres humanos, todos os cuidados éticos foram respeitados. O relato reflete sobre a utilização de uma imagem fixa na coleta de dados, que não foi idealmente representativa aos colaboradores, necessitando de adaptação na metodologia do trabalho. Conclui-se que a utilização da imagem como recurso em pesquisas na saúde permite maior aproximação aos sujeitos da pesquisa, alcançando mais profundamente os seus pensamentos e manifestações.
\end{abstract}

Palavras-chave: Pesquisa qualitativa; Imagem; Saúde bucal; Enfermagem.

\begin{abstract}
Image is an relevant tool in qualitative research. This study aims to describe the experience of using the image as a method of data collection through the Free Word Association Technique in a research on oral health care of hospitalized elderly patients. The research took place in a university hospital located in Salvador, Bahia, Brazil, with the nursing team as collaborator of the study. Due to this research is related to human beings, all ethical caution was respected. The report reflects on the use of a fixed image in the data collection, which was not ideally representative of the researched population, necessitating adaptation in the work methodology. It is concluded that the use of the image as a resource in health research allows a closer approximation to the subjects of the research, reaching deeper their thoughts and manifestation.
\end{abstract}

Keywords: Qualitative research; Image; Oral health; Nursing.

\footnotetext{
${ }^{1}$ Mestre em Enfermagem e Saúde, Universidade Federal da Bahia (UFBA). Universidade do Estado da Bahia (UNEB), Guanambi, Bahia, Brasil. E-mail: eofonseca@uneb.br

${ }^{2}$ Doutora em Enfermagem, Universidade Federal da Bahia (UFBA). Universidade Federal da Bahia (UFBA), Salvador, Bahia, Brasil. E-mail: larissa.pedreira@uol.com.br

${ }^{3}$ Doutora em Turismo, Universidade do Algarve (UAlg). Instituto Politécnico de Beja (IPBeja), Beja, Portugal. E-mail: ana.rodrigues@ipbeja.pt

${ }^{4}$ Mestre em Enfermagem e Saúde, Universidade Federal da Bahia (UFBA). Universidade Federal da Bahia (UFBA), Salvador, Bahia, Brasil. E-mail: robertapgoes@ yahoo.com.br

${ }^{5}$ Mestre em Enfermagem e Saúde, Universidade Federal da Bahia (UFBA). Universidade Federal da Bahia

(UFBA), Salvador, Bahia, Brasil. E-mail: nildetesaude@ yahoo.com.br
} 


\section{Introdução}

O uso do recurso de imagem, como método de coleta de dados em estudos qualitativos, é uma forma de pesquisa na saúde que tem apresentado grande visibilidade, principalmente quando se pretende analisar contextos de vida e ambientes de cuidado.

Ademais, as metodologias visuais têm se firmado também em outras áreas do conhecimento, auxiliando investigações, pelas valorosas possibilidades de coleta de dados, permitindo melhor compreensão do ambiente de interesse. Entretanto, a despeito disso, ainda há escassez de referências sobre essa técnica de coleta (CAMPOS, 2011).

A utilização de elementos visuais, como parte do corpus da investigação, revelase como uma importante ajuda ao pesquisador, pois permite coletar informações relevantes capazes de oferecer repostas à questão de pesquisa, ampliando a possibilidade de compreensão do fenômeno (RODRIGUES; COSTA, 2017). Logo, o uso da imagem (dados visuais) assume um papel complementar à utilização de palavras (dados textuais), acrescentando assim valor à investigação, uma vez que evocam ideias, pensamentos e opiniões, muitas vezes “ocultas”. Conforme Rodrigues e Costa (2017), os dados visuais podem ser considerados como corpus e unidade de análise, muitas vezes complementando os dados textuais.

Em pesquisas qualitativas, o discurso dos participantes pode ser complementado com a utilização de imagens, embora esta, por si só, já permita um grande número de informações, visto que possibilita uma variação de conteúdo a depender do observador, de quem produziu a imagem, e da ferramenta utilizada para essa construção (VELLOSO; GUIMARÃES, 2013). No campo da enfermagem, este recurso tem sido amplamente utilizado, conforme uma revisão de escopo com o objetivo de mapear o uso da fotografia como técnica de coleta de dados nas pesquisas qualitativas da área da saúde (ALVES et al., 2019).

De forma complementar, a Técnica de Associação Livre de Palavras (TALP) é também um método de coleta de dados que pode ser utilizado em variadas pesquisas, tendo como suporte o recurso de imagens e figuras, como um motivador para a evocação das palavras. A TALP atende a estrutura psicológica dos indivíduos, por meio de estímulo, sendo bem difundida em pesquisas da psicologia social. Possui uma característica projetiva, onde o colaborador responde aos estímulos por meio de induções captadas de uma palavra ou imagem, gerando informações aparentemente ocultas, 
demonstrando a personalidade e as ideias do sujeito sobre um objeto indutor (NEVES et al., 2014).

A utilização do recurso de imagens em pesquisas qualitativas deve ser planejada e fundamentada no objeto investigado, alinhada ao contexto onde este está inserido. Ademais, o dado visual vem ganhando importância, sendo um campo de estudo cientificamente reconhecido, e com potencial para ser cada vez mais utilizado (ROSE, 2016). Entretanto, para que possa colaborar com a investigação, a forma de exploração da imagem deve ser bem analisada, visto que o seu uso deve levantar compreensões sobre o contexto histórico dos envolvidos na pesquisa (VELLOSO; GUIMARÃES, 2013).

Diante dessas colocações, o relato em tela objetiva descrever a experiência do uso da imagem em uma coleta de dados, por meio da Técnica de Associação Livre de Palavras, em uma pesquisa sobre cuidados com a saúde bucal de pessoas idosas hospitalizadas.

Neste trabalho, optou-se pelo uso da imagem, pois, na prática clínica, observa-se certa "aversão" dos profissionais de saúde com a cavidade bucal da pessoa idosa hospitalizada. Dessa forma, buscou-se trazer evidências mais fortes a respeito de tal observação.

\section{Metodologia}

Trata-se do relato de experiência, produto de um estudo apresentado no $8^{\circ}$ Congresso Ibero-Americano em Investigação Qualitativa, realizado em julho de 2019, em Lisboa (FONSECA et al., 2019a). O relato versa sobre o planejamento e a etapa da coleta de dados por meio da TALP, utilizando-se de uma imagem como indutora da percepção dos participantes a respeito da cavidade bucal de pessoas idosas hospitalizadas. Esse relato é recorte de uma dissertação de Mestrado Acadêmico, apresentada ao Programa de Pós-graduação em Enfermagem e Saúde da Universidade Federal da Bahia (UFBA), situada em Salvador, Bahia, Brasil, intitulada "O cuidado de enfermagem à saúde bucal do idoso hospitalizado".

A coleta de dados foi realizada em um hospital público universitário de Salvador. Este cenário foi escolhido por ser um hospital-escola vinculado ao programa de graduação e pós-graduação da UFBA, com atendimento a uma população de variadas regiões do estado da Bahia. Dentre a população atendida, muitos são pessoas idosas, com baixo nível sócio econômico e educacional, apresentando condições precárias de saúde bucal. 
Constituiram-se em lócus do estudo duas unidades de terapia intensiva da instituição, com demandas clínicas e cirúrgicas, e uma unidade de internamento com perfil de atendimento de clínica médica a pacientes adultos. Justifica-se a utilização destes setores por apresentarem importante número de idosos acamados e em situações de fragilidade com alteração da sua autonomia. Essas alterações podem ser desencadeadas pela senescência, mas, principalmente, pelos longos períodos no leito, assim como pelo uso de dispositivos hospitalares com impedimento de acesso do idoso ao banheiro para ações de higiene corporal, dentre eles a higiene oral, tornando-os, muitas vezes, dependentes da equipe de enfermagem.

Os participantes do estudo foram os membros da equipe de enfermagem atuantes na assistência direta ao público alvo e que atendiam aos critérios de inclusão: ser enfermeiro ou técnico de enfermagem; estar em escala de trabalho nas unidades lócus no período da coleta; e realizar ações de saúde bucal a pessoas idosas internadas (FONSECA et al., 2019a).

$\mathrm{O}$ acesso aos profissionais participantes do estudo foi possível após consulta às escalas de serviço dos mesmos fornecidas pela coordenação de enfermagem das unidades. Os elegíveis, conforme as informações fornecidas, foram convidados pelos pesquisadores, a participar, por meio de contato pessoal no ambiente de trabalho. Houve a recusa de dois técnicos de enfermagem, ambos da unidade de terapia intensiva (FONSECA et al., 2019a).

Para a coleta, foram agendados encontros individuais, em horário previamente combinado, na própria unidade de trabalho do profissional, em local reservado. Foi utilizado, primeiramente, um formulário com questões de caracterização sobre a idade, o tempo de atuação na enfermagem e na unidade lócus, o gênero, a categoria profissional e sobre a realização ou não de alguma capacitação na temática de higiene bucal. Todos os questionamentos foram respondidos verbalmente.

Posteriormente, ainda neste encontro, foi entregue um segundo formulário, contendo instruções sobre a TALP.

O roteiro de orientações destacava que o colaborador deveria realizar a TALP o mais breve possível, e que seriam ofertados no máximo 20 segundos para análise da imagem apresentada, iniciando o processo assim que o participante demonstrasse para a pesquisadora, que estaria pronto. Neste instatante, a pesquisadora verbalizaria a palavra "iniciada" para de fato começar a TALP, apresentando a imagem. Posteriormente, o colaborador deveria escrever cinco palavras isoladas que viessem à mente durante os 20 
segundos, enumerando estas em ordem de importância, sendo que a de número um seria a mais importante.

Anteriormente à coleta, foi realizado um teste piloto com 24 enfermeiras do programa de residência de enfermagem do hospital selecionado, que atuavam nos setores onde seria realizado o estudo.

Utilizou-se para isso outra imagem (fotografia de uma seringa), para observar se houve compreensão das orientações da TALP e, posteriormente, foi apresentada a mesma imagem deste estudo (fotografia de uma prótese dentária) como forma de testagem do instrumento de coleta, a fim de observar a necessidade de ajustes. Entretando, não houve nenhuma dúvida quanto à aplicação do instrumento (TALP), por estas participantes, ou qualquer observação referente à fotografia da prótese utilizada.

Foram aplicadas duas TALPs no estudo: a primeira por meio de uma frase, e outra com o uso da imagem. Utilizou-se um estímulo verbal, por meio da frase previamente elaborada: "Diante de um idoso hospitalizado, o que vem à sua mente quando falo cavidade bucal?". Em seguida, foi apresentada a imagem de uma prótese dentária (Figura 1), impressa em papel A4 com tinta colorida.

A recolha dos dados ocorreu entre setembro e outubro de 2018, e o planejamento para a escolha da imagem a ser utilizada na TALP, foi realizado nos três meses anteriores. A escolha da imagem foi feita com base em leituras, por meio de referências bibliográficas sobre o tema, para alcance de informações sobre a melhor imagem a ser utilizada (FONSECA et al., 2019a).

Foram ainda averiguadas imagens livres em sites da internet, que melhor representassem a cavidade bucal da pessoa idosa hospitalizada. Logo, o rastreio realizouse em sites de busca, utilizando-se de filtro com a seleção da opção de imagens de licenças gratuitas ou de domínio público, por meio das palavras-chave "denture" e "prothesys dental'. Desta busca, não foram identificadas imagens oportunas para a coleta. Partiu-se, então, para sites de compra de imagens, utilizando-se das mesmas palavras-chave, onde foi encontrada a imagem utilizada, conforme figura abaixo. 
Figura 1: Imagem fixa selecionada para a realização da TALP no estudo

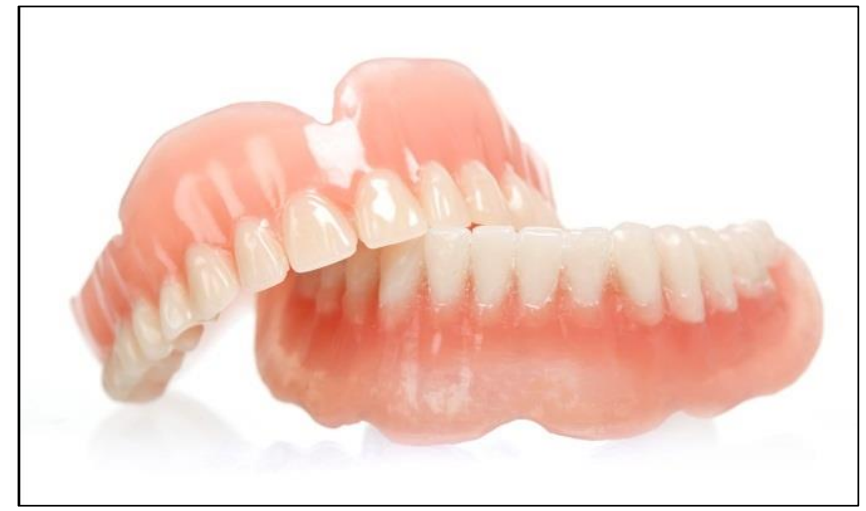

Fonte: sasho/istok.com

O relato de experiência em tela foi embasado no diário de campo da pesquisadora principal, utilizado no momento da coleta de dados, onde foram feitos registros das observações sobre o instante da apresentação da imagem e das respostas dos colaboradores. Ademais, foram registradas impressões deste momento, baseadas também na vivência da pesquisadora enquanto enfermeira assistencial.

O projeto matriz do qual esse estudo faz parte, é intitulado "Cuidado a pessoa idosa durante a hospitalização e transição hospital-domicílio" e, tratando-se de uma pesquisa com seres humanos, foram respeitadas as determinações da Resolução de $\mathrm{n}^{\circ}$ 466/2012 do Conselho Nacional de Saúde (BRASIL, 2012) e a resolução nº 510/2016 (BRASIL, 2016). O projeto foi submetido e aprovado pelo Comitê de Ética e Pesquisa da Escola de Enfermagem da Universidade Federal da Bahia, e do Hospital Universitário Professor Edgard Santos, com Cadastro de Apresentação e Apreciação Ética de número 87976818.6.0000.5531. Os colaboradores que aceitaram participar voluntariamente da pesquisa assinaram um Termo de Consentimento Livre e Esclarecido. Foram preservados os nomes dos participantes, de forma a garantir o anonimato de seus relatos.

\section{Resultados e Discussão}

Participaram do estudo 100 profissionais da área da enfermagem, sendo 45 enfermeiros e 55 técnicos de enfermagem (FONSECA et al., 2019a).

No momento de realização da TALP, referente ao estímulo verbal, não houve qualquer dúvida sobre a evocação. Entretanto, ao realizar o estímulo por meio da imagem fixa, durante as coletas iniciais, os profissionais já pontuavam que a imagem não se relacionava com a imagem cotidianamente percebida em sua prática clínica. 
É bastante significativo que as pesquisas de representação social utilizem conteúdos verbais e de imagem, para alcançar melhor compreensão do sentido da representação (MEDINA FILHO, 2013). Destaca-se que o conteúdo da frase e da imagem apresentam aspectos diferentes na representação social, entretanto, no artigo em tela, não foi objetivo de discussão essa relação, mas sim a reflexão sobre a escolha da melhor imagem a ser apresentada.

Foi possível observar no diário de campo, que a pesquisadora registrou as seguintes falas relatadas pelos profissionais, ao se depararem com a imagem: "Isto [a imagem] não temos aqui"; "É muito limpinho para a nossa realidade [risos]"; "Que linda imagem não? Definitivamente não é o que temos aqui"; "Maravilhoso". As falas revelam que a imagem selecionada não representava a realidade vivenciada pelos profissionais, fato que pode interferir na compreensão do objeto investigado (FONSECA et al., 2019a).

Atentar-se ao diário de campo, permitiu observar que, na imagem utilizada, havia algum elemento que destoava do cotidiano dos colaboradores da pesquisa. Dessa forma, se destaca a importância do olhar crítico e observador do pesquisador e o quanto este elemento metodológico de escrita científica é importante para a fase de análise e de planejamento do trabalho. O diário permite registrar o que se ouve, vê, sente e experiencia no trabalho de campo; sendo um dispositivo de informação que, se utilizado, por meio da criação de notas mentais ou com o uso de palavras chaves, é um recurso de grande ajuda na pesquisa que envolve a investigação, a reflexão e a criatividade (OLIVEIRA, 2014).

A imagem fixa (Figura 1) foi selecionada, a partir de reflexões relacionadas ao fato de que a prótese dentária é comumente manipulada pela equipe de enfermagem, durante o cuidado a cavidade bucal dos idosos hospitalizados. Assim, foi selecionada a imagem de uma prótese dentária total superior e inferior, intencionando evocar tal situação (FONSECA et al., 2019a). A prevalência de edentulismo é alta no Brasil, principalmente na região Norte/Nordeste, onde muitos idosos possuem ausência de todos os dentes (SILVA; OLIVEIRA; LELES, 2015).

O aspecto da prótese dentária apresentada foi de limpa e nova, visando não influenciar sentimentos de aversão a esta. A não utilização de imagens com dentes estragados ou sujos teve a intenção de evitar a associação de tal imagem, a uma possível repulsa da cavidade bucal dos idosos pelos participantes.

Uma pesquisa de revisão sistemática com metanálise, que teve como objetivo principal identificar e sintetizar as evidências sobre barreiras e facilitadores identificados por profissionais prestadores de cuidados bucais a residentes de casas de repouso, 
observou falta de evidências robustas sobre o tema, e aponta a importância em se melhorar o conhecimento dos profissionais de saúde sobre os cuidados com a cavidade bucal, visto a negligência destes com relação a tal cuidado (HOBEN et al., 2017).

Em um trabalho realizado em enfermarias geriátricas na França, Bélgica e Suiça com o objetivo de compreender as dificuldades no processo de higiene oral dos cuidadores de idosos, ancorado em conceitos multivariados, demonstrou-se que há três fortes aspectos: organizacionais, inadequação de treinamento, educação e conhecimento e, ainda, comportamentos, percepções e equívocos dos cuidadores sobre cuidados bucais em idosos (CHAMI et al., 2012). Este estudo desenhou uma "teoria fundamentada em três engrenagens de intertravamento", que apresentou uma das princiapis ferramentas, denominada engrenagem no texto, como motivação para a não realização do cuidado oral: os sentimentos contínuos de "aversão" dos cuidadores, onde foi relatato o sentimento de "nojo" ao visualizar comida e saliva na boca dos idosos, ressaltando que os cuidadores não olhavam dentro da boca dos idosos, assim como seus supervisores, quando avaliavam o seu trabalho (CHAMI et al., 2012).

Esta questão da repulsa ao cuidado oral é significativa quando se reflete na motivação de não realização de uma atividade tão rotineira na enfermagem e implica ainda em ações destorcidas na prática desses profissionais, possibilitando, um cuidado com lacunas, que pode gerar impacto na saúde de idosos. Destarte, a aversão ao cuidado oral foi a principal justificativa de não se utilizar na pesquisa da qual esse relato faz parte, o uso de uma imagem de prótese dentária com sinais de sujidade. Um outro trabalho que corrobora com esta discussão, foi um estudo realizado no Brasil com objetivo de discutir a percepção da equipe de enfermagem sobre a higiene oral de pacientes dependentes hospitalizados, que revelou que o cuidado oral estava sendo realizado pela equipe de enfermagem não como uma necessidade ao cliente, mas sim ao profissional de enfermagem, uma vez que houve o relato do incômdo diante de um paciente com presença de mal odor bucal, onde se observa uma ideia errônea da finalidade do cuidado oral (PASSOS et al., 2014).

No estudo matriz, do qual esse relato é recorte, foi observado que a equipe de enfermagem interage com variadas ações de cuidado direcionado a prótese dentária, desde a sua higienização, até o armazenamento. Entretanto, identificaram-se também lacunas nesse cuidado, como o insuficiente registro das ações realizadas, e divergências relacionadas tanto ao cuidado, como ao acondicionamento deste instrumento (FONSECA 
et al., 2019b). Logo, a familiaridade da equipe de enfermagem com o cuidado a prótese dentária, foi algo que motivou a pesquisadora na escolha da imagem.

Ressalta-se que o uso da TALP nesse trabalho teve como objetivo auxiliar a compreensão sobre o significado da boca de idosos hospitalizados e da prótese dentária para a equipe de enfermagem. Na literatura referente às práticas de higiene bucal, estão descritos variados outros motivos que levam a não realização deste procedimento, por cuidadores de idosos ou equipe de enfermagem, como pouco tempo da equipe para as atividades de cuidado, déficit de recursos humanos e de recursos materiais disponibilizados para ofertar este cuidado (CHAMI et al., 2012; GIBNEY et al., 2015; HOBEN et al., 2017).

A experiência aqui destacada possibilitou compreender a importância do uso da imagem como complemento à coleta de dados, principalmente no sentido de apreender o contexto e o cenário envolvido no objeto de investigação. Por isso, a seleção da imagem necessita ser bem planejada, estando o mais próximo possível do constructo da população abordada.

Ressalta-se que o pesquisador, sendo um ser humano com suas experiências e vivências, também apresenta influência nesta escolha necessitando compreender que as suas experiências são válidas para a pesquisa no que tange principalmente ao seu planejamento e análise. Porém, é relevante compreender também que o cenário e a população na qual se realiza a pesquisa, da mesma forma, influenciam nos elementos de planejamento do trabalho, uma vez que não estando claro algum elemento da pesquisa, pode-se inviabilizar um estudo. Ao se eleger uma imagem ou fazer uma fotografia, o pesquisador e /ou fotógrafo está criando uma moldura para selecionar ideias, uma vez que chamar atenção e direciona a atenção simultaneamente e, dessa forma compõe um significado que se quer refletr em um estudo (ROGER; BLOMGREN, 2020).

A seleção das fontes de imagens a serem utilizadas, necessita ser bem fundamentada e planejada, bem como o processo de escolha destas imagens, a fim de validar a utilização do dado visual, como forma de extração da informação. A influência do pesquisador pode ser exemplificada, quando se descreve a ação de um paparazzo, ao considerar um triunfo fotografar alguma celebridade que tenta se proteger contra a violência visual; intuitivamente, este fotografa seu ego em um corpo alheio. Tal situação, analisada sociologicamente, mostra a mentalidade do fotógrafo, e não, fundamentalmente, o documento da pessoa fotografada (VELLOSO; GUIMARÃES, 2013). 
Alguns questionamentos e reflexões podem ser realizados pelo pesquisador para ajudar na escolha da imagem: qual material seria adequado para tratar o objeto investigado? Quais critérios serão utilizados para esta seleção? Além disso, ao se fazer um inventário da imagem com descrição de todos os seus elementos, reflita sobre o que esses elementos conotam, e quais conhecimentos culturais a imagem se refere e por qual meio são interpretadas (BAUER; GASKELL, 2002).

Reafirma-se, dessa forma, que o pesquisador deve buscar o distanciamento da sua formação cultural, pensando/utilizando, principalmente, o constructo baseado no cotidiano dos participantes em seu cenário. Para isso, é preciso conhecer de perto a população pesquisada e o contexto do objeto. Ao se observar uma imagem, realiza-se uma atividade interpretativa, pois há uma interação do leitor com a fotografia e, a partir desta, este dá sentido ao que visualiza, conforme suas experiências, conhecimentos alcançados e prominência cultural (BAUER; GASKELL, 2002).

Após a experiência descrita, a pesquisadora compreendeu que, a despeito da sua vivência com o objeto, com a reflexão da necessidade de não interferir nas respostas dos colaboradores, ainda, por julgar que há bastante repulsa à prótese dentária na prática clínica, deixou a imagem distante da realidade que se apresenta no ambiente hospitalar, o que influenciou negativamente na coleta e na apreensão da realidade investigada.

O uso da foto-elicitação, apesar de ser comum em trabalhos nas áreas sociais, se pontua com lacunas no rigor científico (SRESNEWSKY et al., 2018). Dessa forma, é interessante que o processo de escolha da imagem que será utilizada na pesquisa, seja embasado em variados elementos e critérios de seleção, sendo fundamental dispensar cuidados no detalhamento metodológico ao se utilizar imagens nos estudos, para que as novas pesquisas possam propor novas aplicações.

Mesmo compreendendo que a escolha da imagem requer discussões aprofundadas relacionadas com o objeto da pesquisa, muitas vezes elas não recebem atenção pronunciada nas publicações, onde também pouco se detalha o motivo da sua escolha. Compreende-se assim a importância dos dados visuais, contudo, o seu processo de criação e compartilhamento precisam ser rigorosos e melhor detalhados (ROGER; BLOMGREN, 2020).

Sobre isso, autores pontuam que em métodos criativos, destacando-se aqui o uso de imagens, reflexões e discussões do cotidiano são incentivadas, aumentando o envolvimento na recolha de dados pelos pesquisadores junto ao público pesquisado. 
Porém, ainda existem poucas discussões metodológicas sobre tal abordagem (BROOKS; LAINIO; LAŽETIĆ, 2020; ROGER; BLOMGREN, 2020)

Ademais, a elicitação não é um ato único, mas sim um processo e uma mentalidade que são importantes em todas as etapas da pesquisa. $O$ uso da imagem pode ser considerada como uma ponte para a comunidade entrar no meio acadêmico, mas também uma forma da academia se estender para a comunidade, refletindo nesta ligação sobre o que querem ou não descobrir a respeito do objeto de estudo (ROGER; BLOMGREN, 2020).

Vale lembrar que, neste estudo, foi realizado um teste piloto com 24 enfermeiras residentes de terapia intensiva. Contudo, no momento desta avaliação, não se observou falta de representatividade da imagem neste grupo, ou alguma referência de que a imagem não estaria próxima da realidade, possivelmente pela pouca experiência das residentes ainda no início do curso, no cuidado com a saúde oral das pessoas idosas hospitalizadas.

Logo, destaca-se a singularidade da pesquisa qualitativa, reafirmando que nesta, sempre há a necessidade de avaliar e reavaliar as situações conforme o objeto de investigação, de modo a encontrar o melhor caminho para a coleta e interpretação dos dados. Este movimento é característico desse tipo de pesquisa, e exige esforço metodológico, visto que apresenta questões subjetivas que necessitam de uma análise criteriosa e sistemática (MINAYO, 2012).

Dessa forma, o acesso às imagens deve ser cuidadoso e a sua utilização requer ações prévias de modo a ler e entender as figuras selecionadas. Tais atitudes são das mais importantes em pesquisas com o uso desse recurso (MEDINA FILHO, 2013). Ao se utilizar o método fotográfico, por exemplo, em uma coleta de dados, existem vantagens como mais possibilidades de reflexões dos participantes, uma menor formalidade nas entrevistas e maior percepção de impressões subjetivas. Porém, o uso deste recurso pode ocasionar dificuldades relacionadas aos aspectos operacionais, questões éticas e incertezas quanto à profundidade da análise (ALVES et al., 2019).

Autores também apontam que o uso da imagem para coleta de dados, permite uma análise de ideias e opiniões dos colaboradores da pesquisa, possibilitando mais respostas à questão de investigação, na medida em que proporciona fluidez na comunicação entre pesquisador e colaboradores (RODRIGUES; COSTA, 2017). O uso, por exemplo, da fotoelicitação, acentua o grau de intimidade entre pesquisador e colaborador. Por isso, as imagens devem se apresentar em um contexto, exigindo do pesquisador constantes narrativas internas e externas sobre o que se pretende retratar (BANKS, 2013). 
A experiência aqui relatada gerou a compreensão e a necessidade de adaptação para uma nova imagem, a partir do entendimento da primordialidade de aproximação ao colaborador da pesquisa, reafirmando a subjetividade do método qualitativo e, por isso, a importância do pesquisador se atentar a todas as informações, com um olhar crítico e inquiridor.

Ademais, é importante ter em vista que a análise interpretativa dos dados nas ciências sociais, não é apresentada de forma independente, mas de forma colaborativa, entre o pesquisador e o colaborador da pesquisa. Assim, assegura-se que o pesquisador alcance o melhor acesso aos pensamentos, palavras e atos dos colaboradores e fenômenos investigados (BANKS, 2013).

Diante da experiência aqui relatada, o resultado oriundo da imagem apresentada, não foi considerado na dissertação, que utilizou o material recolhido das entrevistas, a partir de um questionário elaborado previamente. Assim, a imagem selecionada e utilizada será revista, para coleta em outro desdobramento da pesquisa. Sendo relevante expor a experiência em tela a fim de contribuir com a reflexão durante a escolha da imagem para coleta de dados por parte dos pesquisadores em outros cenários de estudo.

\section{Conclusão}

Na pesquisa qualitativa, a utilização de diferentes formas de coleta como imagens, falas e observação enriquece a pesquisa, possibilitando uma análise mais profunda e situada do fenômeno. Para tanto, é preciso rigor na seleção, no planejamento e na organização dos instrumentos de coleta, com foco no objeto a ser investigado.

$\mathrm{Na}$ área da saúde, o recurso da imagem pode oferecer maior aproximação aos colaboradores e seu contexto, permitindo um mergulho mais intenso, além de melhor compreensão de seus pensamentos e expressões.

A partir dessa experiência, foi possível discutir a necessidade de se reavaliar o uso da ferramenta na recolha de dados utilizada e pretende-se repensar e aprofundar a utilização desta imagem para produção de nova coleta, dando continuidade aos estudos com a temática.

\section{Referências}

ALVES, K. et al. Fotografia como técnica de coleta de dados nas pesquisas qualitativas da área da saúde: scoping review. In: CONGRESSO IBERO-AMERICANO DE INVESTIGAÇÃO QUALITATIVA, 8., 2019. Lisboa. Anais... Aveiro: Ludomedia, 2019. p. 154-163. Disponível 
em: https://proceedings.ciaiq.org/index.php/CIAIQ2019/article/view/2014. Acesso em: 13 fev. 2020.

BANKS, M. Dados visuais para pesquisa qualitativa. Porto Alegre: Artmed, 2013.

BRASIL. Ministério da Saúde. Conselho Nacional de Saúde. Resolução nº 466, 12 de dezembro de 2012. Brasília - DF, Brasil: Ministério da Saúde, 2012. Disponível em: https://bvsms.saude.gov.br/bvs/saudelegis/cns/2013/res0466_12_12_2012.html. Acesso em: 13 fev. 2020.

BRASIL. Ministério da Saúde. Conselho Nacional de Saúde. Resolução n 510, 7 de abril de 2016. Brasília - DF, Brasil: Ministério da Saúde, 2016. Disponível em: http://bvsms.saude.gov.br/bvs/saudelegis/cns/2016/res0510_07_04_2016.html. Acesso em: 13 fev. 2020.

BAUER, M.; GASKELL, G. Pesquisa qualitativa com texto, imagem e som: um manual prático. Petrópolis: Vozes, 2002.

BROOKS R.; LAINIO; LAŽETIĆ, P. Using creative methods to research across difference. An introduction to the special issue. International Journal of Social Research Methodology, Londres, v. 23, n. 1, p. 1- 6, oct. 2019. Disponível em: http://10.1080/13645579.2019.1672281. Acesso em: 23 fev. 2020.

CAMPOS, R. B. C. Imagem e tecnologias visuais em pesquisa social: tendências e desafios. Análise Social, [S.I.], v. XLVI, n. 199, p. 237-259, 2011.

CHAMI, K. et al. Reluctance of Caregivers to Perform Oral Care in Long-Stay Elderly Patients: The Three Interlocking Gears Grounded Theory of the Impediments. Journal of the American Medical Directors Association, Canadá, v. 13, n. 1, p. e1-e4, jan. 2012. Disponível em: https://www.ncbi.nlm.nih.gov/pubmed/21752721. Acesso em: 13 fev. 2020.

FONSECA, E.O.S. et al. O uso da imagem em pesquisa qualitativa imagem em pesquisa qualitativa na área da saúde: relato de experiência. In: CONGRESSO IBERO-AMERICANO DE INVESTIGAÇÃO QUALITATIVA, 8., 2019. Lisboa. Anais... Aveiro: Ludomedia, 2019a. p. 1408-1413. Disponível em: https://proceedings.ciaiq.org/index.php/CIAIQ2019/article/view/238. Acesso em: 13 fev. 2020.

FONSECA, E.O.S. et al. O cuidado de enfermagem no acondicionamento da prótese dentária de idosos hospitalizados. Acta paul. enferm., São Paulo, v. 32, n. 4, p. 442-448, aug. 2019b. Disponível em: https://doi.org/10.1590/1982-0194201900060. Acesso em: 23 fev. 2020.

GIBNEY, J. et al. Nurses' knowledge, attitudes, and current practice of daily oral hygiene care to patients on acute aged care wards in two Australian hospitals. Special Care in Dentistry, Toronto, v. 35, n. 6, p. 285-293, nov. 2015. Disponível em: https://www.ncbi.nlm.nih.gov/pubmed/26297474. Acesso em: 04 mai. 2019.

HOBEN, M. et al. Barriers and facilitators in providing oral care to nursing home residents, from the perspective of care aides: A systematic review and meta-analysis. International Journal of Nursing Studies, Waltham v. 73, p. 34-51, ago. 2017. Disponível em: https://www.ncbi.nlm.nih.gov/pubmed/28531550. Acesso em: maio. 2019.

MEDINA FILHO, A. L de. Importância das imagens na metodologia de pesquisa em psicologia social. Psicologia \& Sociedade, Belo Horizonte, v. 25, n. 2, p. 263-271. 2013. Disponível em: http://www.scielo.br/pdf/psoc/v25n2/03.pdf. Acesso em: maio. 2019. 
MINAYO, M. C. S. Análise qualitativa: teoria, passos e fidedignidade. Ciência \& Saúde Coletiva, Rio de Janeiro, v. 17, n. 3, p. 621-626, mar. 2012. Disponível em: http://www.scielo.br/scielo.php?script=sci_arttext\&pid=S1413-81232012000300007. Acesso em: maio. 2019.

NEVES, D. A. B. et al. Protocolo verbal e teste de associação livre de palavras: perspectivas de instrumentos de pesquisa introspectiva e projetiva na ciência da informação. PontodeAcesso, Salvador, v. 8, n. 3, p. 64, 20, jan. 2014. Disponível em: https://portalseer.ufba.br/index.php/revistaici/article/view/12917/9240. Acesso em: maio. 2019.

OLIVEIRA, R.C.M. (Entre)linhas de uma pesquisa: o diário de campo como dispositivo de (in)formação na/da abordagem (auto)biográfica. Revista Brasileira de Educação de Jovens e Adultos, Salvador, v. 2, n. 4, p. 69-87. 2014. Disponivel em: https://www.revistas.uneb.br/index.php/educajovenseadultos/article/view/1059. Acesso em: 27 fev. 2020.

PASSOS, S.S.S. et al. Oral hygiene to a hospitalized dependent patient: perceptions of a nursing team. Revista de Pesquisa Cuidado é Fundamental Online, Rio de Janeiro, v. 6, n. 4, p.13961408, out/dez. 2014. Disponível em: https://www.redalyc.org/articulo.oa?id=505750770008. Acesso em: 22 fev. 2020.

RODRIGUES, A.I.; COSTA, A. P. A imagem em investigação qualitativa: análise de dados visuais. In: AMADO, J.; CRUSOÉ, N.M.C. (Org.). Referenciais Teóricos e Metodológicos de Investigação em Educação e Ciências Sociais. Salvador: Edições UESB, 2017. p. 195-21.

ROSE, G. Visual Methodologies: An Introduction to Researching with Visual Materials. 4. ed. Londres: S. Publications, 2016.

ROGER, K.S.; BLOMGREN, C. Elicitation as a Mind-Set: Why Visual Data

Matter? International Journal of Qualitative Methods, Canadá, v. 2, n. 18, p. 1-9, mar. 2019. Disponível em: https://doi.org/10.1177/1609406919835378. Acesso em: 23 fev. 2020.

SILVA, E.T.; R.T.; OLIVEIRA; LELES, C.R. O edentulismo no Brasil: epidemiologia, rede assistencial e produção de próteses pelo Sistema Único de Saúde. RevistaTempus Actas de saúde Colet, Brasília, v. 3, n. 9, p. 121-134, set. 2015. Disponível em:

http://www.tempusactas.unb.br/index.php/tempus/article/view/1790. Acesso em: 02 maio. 2019.

SRESNEWSKY, K.B.G.B. et al. O Uso da Foto-Elicitação nas Pesquisas em Marketing.

Brazilian Journal of Marketing, São Paulo, v.17, n.6, p. 839-848, nov. 2018. Disponível em: https://doi.org/10.5585/bmj.v17i6.3748. Acesso em: 23 fev.2020.

VELLOSO, M. P.; GUIMARÃES, M. B. L. A imagem na pesquisa qualitativa em saúde. Ciência \& Saúde Coletiva, Rio de Janeiro, v. 18, n. 1, p. 245-252, jan. 2013. Disponível em: http://www.scielo.br/scielo.php?pid=S1413-81232013000100025\&script=sci_abstract\&tlng=pt. Acesso em: 02 maio. 2019.

Recebido em: 10 de fevereiro de 2020.

Aceito em: 15 de março de 2020. 